\title{
Blood Culture of Neonates in Paediatric Department and their Antimicrobial Susceptibility Pattern in and around Nims University, Jaipur, India
}

\author{
Shilpa Pradhan ${ }^{1 *}$, Joy Chakraborty ${ }^{1}$, Anjali Kulshrestha ${ }^{1}$, \\ Suman Rishi ${ }^{2}$ and Pardeep Goyal ${ }^{3}$
}

\author{
${ }^{1}$ Department of Microbiology, NIMS Medical College, Shobha nagar, Jaipur, India \\ ${ }^{2}$ Department of Medical Microbiology, NIMS Medical College, Shobha nagar, Jaipur, India \\ ${ }^{3}$ NIMS Medical College, Shobha Nagar, Jaipur, India \\ *Corresponding author
}

\begin{abstract}
A B S T R A C T
Blood stream infections are very common in the paediatric age group and these are one of the common causes of morbidity and mortality in neonates and children. Objectives of the

Keywords

Mango pulp, Mango peel powder (MPP),

Nectar, Chemical

preservative,

Sensory properties.

Article Info

Accepted:

15 March 2017

Available Online:

10 April 2017 study are to isolate the different organisms from Blood cultures of Neonates by BACTEC method and know their Antibiotic susceptibility patterns. We processed 100 Blood samples of the Neonates from PICU of NIMS Medical College and Hospital between January and June 2016 (06 months). Samples were collected in BACTEC PEDS PLUS vials with all aseptic precautions. The vials were placed in the BACTEC 9050 and cultured. The positive vials were further plated on BA, MacConkey Agar looked for the growth and identified by necessary biochemical reactions and antibiotic susceptibility is done by Kirby-Bauer method. Out of 100 blood culture samples, 58 were positive. Out of 58, 18 yielded CoNS (31.03\%), 12- Klebsiella spp (20.68\%), 04 - Staphylococcus aureus (6.89\%), 06 - Enterococcus spp (10.34\%), 12- Enterobacter spp (20.68\%), and 02 each of E. coli $(3.44 \%)$, Citrobacter $(3.44 \%)$ and Acinetobacter $(3.44 \%)$. Significantly high rates of Gram Negative bacterial infections were seen followed by Gram Positives. Automated Blood Culture systems significantly reduced the time required for processing of samples and also facilitated the yield of diverse organisms. Antibiotic Susceptibility patterns also showed Gram Negative bacteria to be less sensitive than Gram Positive bacteria.
\end{abstract}

\section{Introduction}

Blood stream infections are very common in the paediatric age group and these are one of the common causes of morbidity and mortality in neonates and children. The incidence of sepsis is increasing all over the world leading to high morbidity and mortality rates (Kavitha et al., 2010). Detection of Bacteremia by blood culture is critical in managing patients with infection, and directs the appropriate selection of antimicrobials. Blood culture is a common laboratory investigation where blood is inoculated into culture medium and continuously monitored for positive growth. Media used in the blood culture bottles support the growth of most medically important bacteria. Manual culture techniques often take a longer duration for detection and isolation of organisms (Tarai et 
al., 2012). Blood culture technology has changed from tube or bottles of liquid culture medium requiring frequent inspection, microscopic or blind plating on to solid culture medium to see if growth has occurred to modern, closed, computer based systems, which assess changes in $\mathrm{CO} 2$ indicating growth every 10-15 minutes (the BacT/ALERT microbial detection system, Organon Teknika Corporation, Durham, North Carolina, USA) (Butterly, 2002). The BacT/ ALERT method is reported to more rapidly detect positive blood culture results in pediatric and newborn patients than other systems (Kumer et al., 2001).

Sepsis is a commonly encountered and potentially life threatening problem in Neonatal intensive care units (NICU's), it is not easy to establish a definite diagnosis of Sepsis in the neonates. Early clinical signs are mostly non-specific and in conclusive (Khadija et al., 2011). Current study was undertaken to find out the common bacterial pathogens and their susceptibility pattern in neonates with sepsis in a tertiary care hospital providing neonatal intensive care services. Improvement in outcome and successful treatment depends on early initiation of appropriate antibiotic therapy (Srinivasa et al., 2014). It is estimated that $26 \%$ of newborn infants who die do so as a result of infections that occur around birth. After the first week of life, infections are the main cause of neonatal death in many countries. Most published data are from hospitals where Klebsiella species, Escherichia coli (E. coli), and Staphylococcus aureus (S. aureus) are the most common causes of infection (Shrestha et al., 2013). The early symptoms of sepsis are non-specific, and the outlook is considered to be worst in babies in whom antibiotics are started late. Antibiotics are therefore generally given to all babies with clinical or laboratory indicators of infection and babies at high risk of early onset sepsis. A large number of babies who are evaluated for sepsis do not have proven infection. This means that most of the antibiotics given to babies on the neonatal unit are given to babies without infection. Inappropriate use of antibiotics has been implicated in the development of multiresistant bacteria in hospitals, Recently published studies suggest that antibiotic courses may be safely reduced to 24-36 hours in asymptomatic term babies (Kumar et al., 2001; Khadija et al., 2011).

Thus the Objective of this study was to measure the time required for the bacteria to be detected in blood cultures taken from suspected sepsis newborns by the BACTEC microbial detection system (Waricha et al., 2006) and also to establish an anti-biogram pattern in and around NIMS University, Jaipur.

\section{Materials and Methods}

NIMS Medical College and Research Centre is a 950 bedded hospital located in Jaipur. There is a dedicated team of 05 doctors at Central Laboratory handling approximately 600-650(includes pathology, microbiology and biochemistry samples) samples per day. Microbiology lab is equipped with BACTEC 9050 Blood Culture System (BD).

In this study we used BACTEC PEDS PLUS vials (Yellow top- paediatric aerobic) from the neonates suspected of Septicaemia from PICU. We analyzed 100 blood cultures collected during January to June 2016 after approval of the Hospital ethical committee.

Blood Collection: Under strict aseptic conditions, disinfect the venepuncture site using chlorhexidine with $70 \%$ alcohol swabs, allowing the site to completely dry. Draw 0.5$1.0 \mathrm{ml}$ of blood and place it in paediatric aerobic bottle. Fill the necessary requisition forms. All the paediatric aerobic bottles were sent to Microbiology laboratory. 
Sample processing, identification and sensitivity

BD BACTEC 9050 System was used for incubation and the bottles were incubated until microbial growth was detected (BACTEC). BACTE 9050 is an automated blood culture system, which contains a sensor which responds to the concentration of $\mathrm{CO}_{2}$ produced by the metabolism of micro organisms or the consumption of $\mathrm{O}_{2}$ needed for the growth of micro organisms. The sensor is monitored by the instrument every ten minutes for an increase in its fluorescence, which is proportional to the increasing amount of $\mathrm{CO}_{2}$ or the decreasing amount of $\mathrm{O}_{2}$ present in the vial. BACTEC 9050 bottles that showed growth were plated onto sheep BA and MacConkey Agar and further incubated at 35+/- $2^{0}$ C. Growth's were stained by Gram's method (WHO; Koneman's Color Atlas and Textbook of Diagnostic Microbiology, $5^{\text {th }}$ edn). The positive growth was further processed by routine biochemical reactions and Antibiotic Susceptibility was put up by modified Kirby Bauer's method (Lalitha, 2004). CLSI guidelines were followed for interpretation of results (Collins et al., 1995; Forbes et al., 2002; Evans et al., 1996; WHO, 1980).

\section{Results and Discussion}

Our study analyzed the blood cultures from $1^{\text {st }}$ January to $30^{\text {th }}$ June 2016. A total of 100 Blood cultures were received from Pediatric ICU. Total blood cultures that came positive were 58. Out of 58, 28 samples yielded Gram Positive cocci (CONS-18, S. aureus-04, and Enterococcus-06) and 30 samples yielded Gram Negative Bacilli (Klebsiella-12, E. coli02, Citrobacter -02, Enterobacter-12 and Acinetobacter-02). Most of the blood cultures grew within $72 \mathrm{hrs}$ of incubation.

Table.1 Antibiotic used in the study

\begin{tabular}{|l|l|l|l|}
\hline S.NO & Antibiotic & Potency & Abbreviations \\
\hline 1. & Amoxiclav & $30 \mathrm{mcg}$ & AMC \\
\hline 2 & Penicillin & $10 \mathrm{Units}$ & P \\
\hline 3 & Linezolid & $30 \mathrm{mcg}$ & LZ \\
\hline 4 & Vancomycin & $30 \mathrm{mcg}$ & VA \\
\hline 5 & Ampicillin & $10 \mathrm{mcg}$ & AMP \\
\hline 6 & Azithromycin & $15 \mathrm{mcg}$ & AZM \\
\hline 7 & Pipercillin/Tazobactum & $100 / 10 \mathrm{mcg}$ & PIT \\
\hline 8 & Cefoperazone/Sulbactum & $30 / 15 \mathrm{mcg}$ & CFS \\
\hline 9 & Cefoxitin & $30 \mathrm{mcg}$ & CTN \\
\hline 10 & Ceftriaxone & $30 \mathrm{mcg}$ & CTR \\
\hline 11 & Chloramphenicol & $30 \mathrm{mcg}$ & C \\
\hline 12 & Ofloxacin & $5 \mathrm{mcg}$ & OF \\
\hline 13 & Cefepime & $30 \mathrm{mcg}$ & CPM \\
\hline 14 & Amikacin & $30 \mathrm{mcg}$ & AK \\
\hline 15 & Imipenem & $10 \mathrm{mcg}$ & IPM \\
\hline 16 & Ceftazidime-Clavulanic acid & $30 / 10 \mathrm{mcg}$ & CAC \\
\hline
\end{tabular}


Table.2 Number of organism isolates from culture positive neonates

\begin{tabular}{|l|l|}
\hline Isolates & Frequency of isolates (\%) \\
\hline CoNS & $18(31.03 \%)$ \\
\hline Klebsiella spp. & $12(20.68 \%)$ \\
\hline S.aureus & $04(6.89 \%)$ \\
\hline Enterococcus & $06(10.34 \%)$ \\
\hline E.coli & $02(3.44 \%)$ \\
\hline Citrobacter & $02(3.44 \%)$ \\
\hline Enterobacter & $12(20.68 \%)$ \\
\hline Acinetobacter & $02(3.44 \%)$ \\
\hline
\end{tabular}

Fig.1 BACTEC 9050 Blood culture system
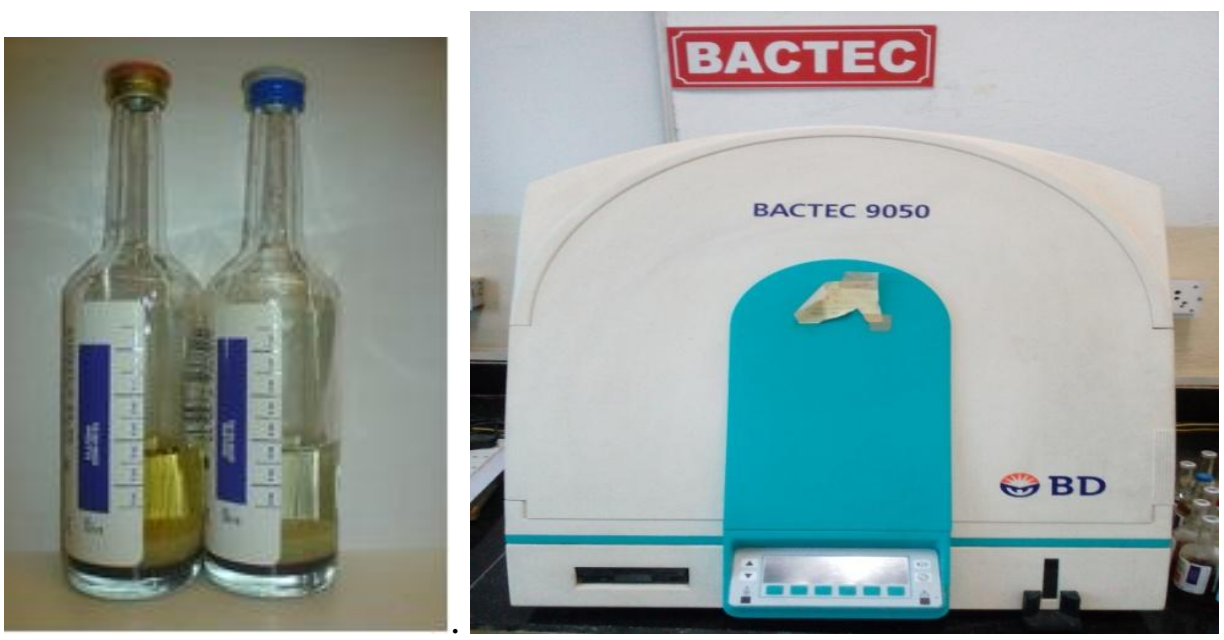

Graph.2 No. of organism isolates from blood culture positive neonates

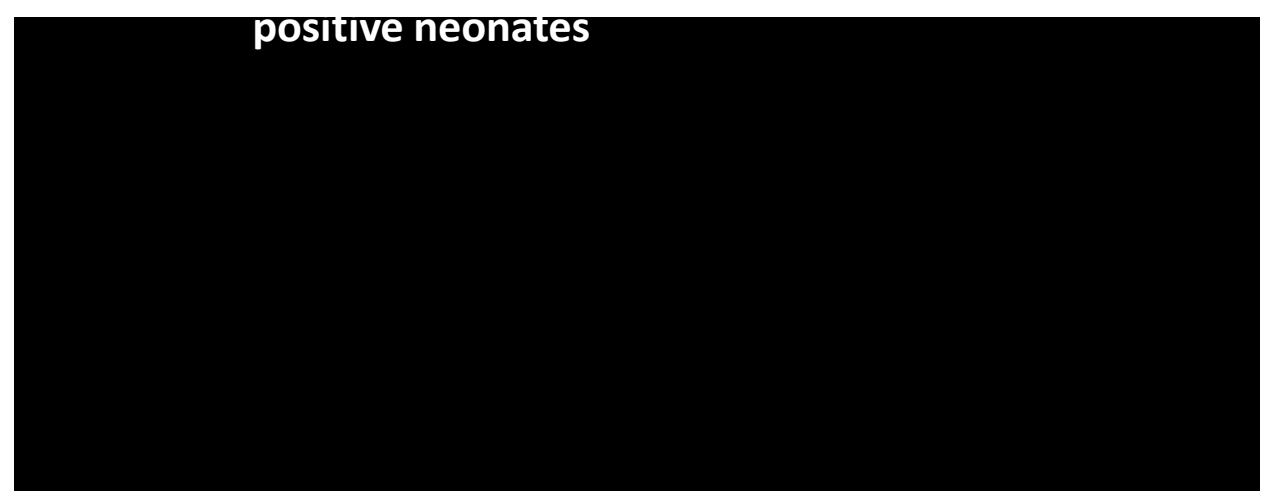


Graph.3 Gram positive and Gram negative organisms in blood sample- total isolates ( $\mathrm{n}=58)$

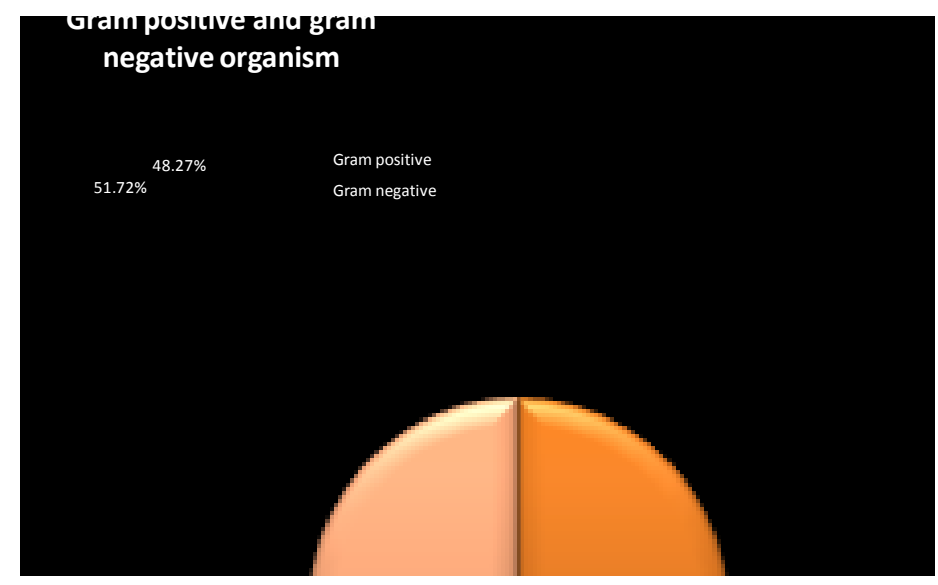

Graph.4 Antibiotic sensitivity patterns of blood sample ( $\mathrm{n}=58)$

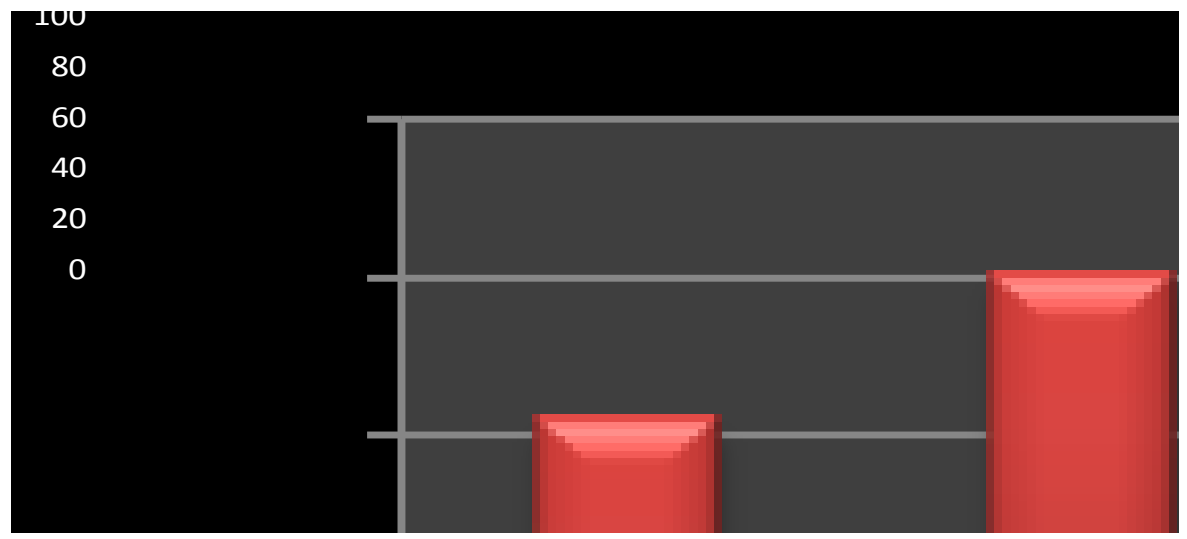

Graph.6 Antibiotic sensitivity of Gram Negative organism in blood sample $(n=30)$

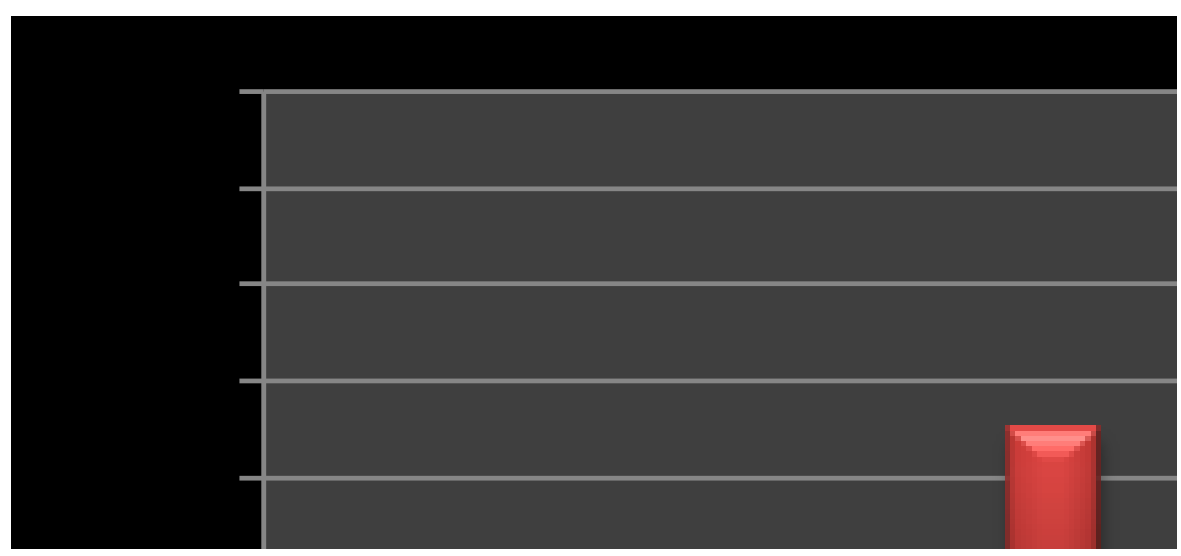


Neonatal septicemia refers to generalized bacterial infection documented by positive blood culture in first five weeks of life. It is the major cause of mortality and morbidity accounting for $30-50 \%$ neonatal deaths in developing countries. In India a lot of neonatal mortality is accountable by septicemia and its treatment failure due to emergence of drug resistance. The fact is that the isolated organisms have developed increased drug resistance over the last few years. Most of the Laboratories in India use conventional Glucose broth/BHI broth for Blood culture routinely. Use of BACTEC PEDS PLUS bottles is intended to maximize the yield of obligate aerobes and anaerobes. BACTEC BD bottles not only yielded Gram Positive cocci, but also Gram Negative bacilli, they also showed positive cultures in patients with single dose of Antibiotics, since the Antimicrobial activity is neutralised by resins present in the bottles. Blood cultures remain a valuable microbiological test for diagnosis of Septicaemia. Automated blood culture methods significantly reduced the time required for processing of samples and also facilitated yield of GPC and GNB.

Neonatal septicemia remains a major clinical problem with high morbidity and mortality rate, especially in developing countries like India. The present study showed that gram negative bacteria were the most prevalent cause of infections in neonates in our hospital. Klebsiella spp. was the most commonly isolated gram negative organism whereas CONS were the most commonly isolated from gram positive organism. Drug resistance to conventional antibiotics is a common problem. As neonatal septicemia is considered a life threatening Emergency condition, prompt treatment with antibiotics is necessary. WHO has recommended the use of pencillin or ampicillin plus an aminoglycoside for neonates. The present study showed Imipenem, piperacillin tazobactum,
Cefaperazone sulbactum and Ceftazidimeclavulunic acid are more effective drug for the treatment of gram negative organisms. Linzeolid, Vancomycin shows $100 \%$ susceptibility for gram positive organisms and more effective drugs for the treatment of gram positive isolates. So, it will important to continue surveillance of neonatal sepsis in order to follow closely changing in trends and risk factor to obtained information for empiric antibiotic therapy and to react rapidly in case of major changes in susceptibility patterns and occurrence of outbreaks.

\section{References}

BACTEC. Fluorescent Series Users Manual. Document Number MA - 0074. BD Biosciences.

Collins, C.H., Lyne, P.M. and Grange, J.M. 1995. Microbiological Methods. Butterworths, London, 94-96.

Evans, E.G.V., Killington, R.A., Heritage, J. 1996. Introductory Microbiology. (Cambridge University Press.)

Forbes, B.A., Sahm, D.F. and Weissfeld, A.S. 2002. Bailey and Scott's Diagnostic Microbiology. 11 th ed (The CV Mosby Company, St. Louis).

Kavitha Prabhu, Sevitha Bhat, Sunil Rao. 2010. Bacteriologic Profile andAntibiogram of Blood Culture Isolates in a Pediatric Care Unit, J. Lab. Physicians, Vol-2 / Issue-2.

Khadija Guerti, Helena Devos, Margareta, M., Ieven and Ludo, M., Mahieu. 2011. Time to positivity of neonatal blood cultures: fast and furious, J. Med. Microbiol., 60: 446-453.

Koneman, E.W., Allen, S.D., Janda, W.M., Schreckenbergu, P.C. and Winn, Jr. W.C. Color Atlas and Textbook of Diagnostic Microbiology. 5 th ed.

Kumar, Y., M. Qunibi, T.J. Neal, C.W. Yoxall. 2001. Time to positivity of neonatal blood cultures, Arch. Dis. 
Child Fetal Neonatal Ed., 85 F182: F182-F186, Downloaded from group.bmj.com on July 27, 2012 Published by fn.bmj.com

Lalitha, M.K. 2004. Manual on Antimicrobial Susceptibility Testing. Christian Medical College, Vellore, pp43.

Shrestha, S., Shrestha, N.C., Dongol Singh, S., Shrestha, R.P.B., Kayestha, S., Shrestha, M., Thakur, N.K. 2013. Bacterial Isolates and its Antibiotic Susceptibility Pattern in NICU, Vol.11 | No. 1 | Issue 41.

Srinivasa, S., Arunkumar, D. 2014. Department of Pediatrics, Kempegowda institute of Medical sciences and research hospital, Bangalore. Bacterial isolates and their Antibiotic susceptibility patterns in Neonatal sepsis, Curr. Pediatr. Res.
Tarai, B., P. Das, D. Kumar, S. Budhiraja. 2012. Comparative evaluation of paired blood culture (aerobic/anaerobic) and single bold culture, along with clinical importance in catheter versus peripheral line at a tertiary care hospital, Indian $J$. Med. Microbiol., Vol-30/Issue -2/ Page: 187-192.

Waricha Janjindamai and Saranwan Phetpisal. 2006. Department of Pediatrics, Faculty of Medicine, Prince of Songkla University, Hat Yai, Songkhla, Thailand, Time to positivity of blood culture in newborn infants, Vol 37 No.

WHO. 1980. Manual of Basic Techniques for a Healthy Laboratory.

WHO. Guidelines on Standard Operating Procedures for Microbiology. Chapter 4: Staining Techniques.

\section{How to cite this article:}

Shilpa Pradhan, Joy Chakraborty, Anjali Kulshrestha, Suman Rishi and Pardeep Goyal. 2017. Blood Culture of Neonates in Paediatric Department and their Antimicrobial Susceptibility Pattern in and around Nims University, Jaipur, India. Int.J.Curr.Microbiol.App.Sci. 6(4): 19401946. doi: https://doi.org/10.20546/ijcmas.2017.604.231 